\title{
Soybean AP2-Domain Transcription Factor GmWRI4 Optimize Ratio of Oleic Acid to Linoleic Acid in Seed
}

Qin Di ( $\sim 603988396 @ q q . c o m$ )

Zhongkai University of Agriculture and Engineering

\section{Angela Piersanti}

University of Camerino

Qi Zhang

Guangzhou University of Chinese Medicine

Cristina Miceli

University of Camerino

Xiaoyi Liu

Guangzhou University of Chinese Medicine

\section{Research Article}

Keywords: Soybean, Linoleic acid, Genome-wide association study, RNA-seq, WRI1

Posted Date: October 29th, 2021

DOI: https://doi.org/10.21203/rs.3.rs-995756/v1

License: (9) (1) This work is licensed under a Creative Commons Attribution 4.0 International License. Read Full License 


\section{Abstract}

Background: Soybean [Glycine max (L.) Merrill] oil is a complex mixture of five fatty acids (oleic, linoleic, linolenic, palmitic and stearic). As a vegetable oils, soybean oil has a less than desirable fatty acid composition. The high content of linoleic acid contributes to poor oil oxidative stability. Soybean oil with lower linoleic acid content is desirable. To investigate the genetic architecture of linoleic acid in soybean seed, 510 soybean germplasms from China were collected as natural populations.

Results: Phenotypic identification results showed that the content of linoleic acid varied from $36.22 \%$ to $72.18 \%$. After a total of 2,423,512 nucleotide polymorphism (SNP) were obtained, a new candidate gene Glyma.04G116500.1 (GmWR/14) related to linoleic acid was discovered by 3-year long Genome-wide association analysis (GWAS). GmWR/14 belongs to the plant WRI1 protein family. The GmWR/14 showed a negative correlation with the linoleic acid content and the correlation coefficient was -0.912. To test whether GmWR/14 can lead to lower linoleic acid content in soybean seed, we introduced GmWR/14 into the soybean genome. Overexpression of GmWR/14 leads to higher accumulation of oil with lower linoleic acid content in soybean seed. RNA-seq verified that GmWR/14-overexpressed lines showed lower accumulation of GmFAD2-1 and GmFAD2-2b than nontransgenic lines.

Conclusions: These results indicate that the down-regulation of FAD2 gene triggered by the transcription factor WR/1 is the underlying mechanism reducing linoleic acid level of seed oil. GmWR/14 is a new key candidate gene related to linoleic acid. These results will significantly improve understanding of the transcriptional control exerted by GmWR/14.

\section{Background}

Soybeans [Glycine max (L.) Merrill] is a major oilseed crop providing edible oil and protein in China(Liu et al., 2020). Soybean oil is a complex mixture of five fatty acids, they are oleic acid (C18:1), linoleic acid (C18:2), linolenic acid (C18:3), stearic acid (C18:0) and palmitic acid (C16:0) (Zhao et al., 2019). Fatty acid composition of soybean oil ranged from about 15-33\% in oleic, $43-56 \%$ in linoleic, $5-11 \%$ in linolenic, and $11-26 \%$ in saturated acids (Zhang et al., 2018). As a food commodity, soybean oil has a less than desirable fatty acid composition (Clemente and Cahoon, 2009). Soybean oil quality is determined by fatty acid composition. Linolenic acid is needed for normal human growth and development (De Groot et al., 2004), and linolenic acid can lower the cholesterol content in the blood (Chan et al., 1991), but linolenic acid is not resistant to high temperature, air oxygen and ultraviolet rays can oxidize linolenic acid, resulting in odor of soybean oil, which is not easy to preserve and lower the nutritional value of soybean oil (Maskan and Karataş, 1998; Tompkins and Perkins, 2000; L. Yang et al., 2009). Linoleic acid belongs to polyunsaturated fatty acids, it is known to have beneficial properties to health (Tompkins and Perkins, 2000), but the oxidative stability values of the oil enriched with linoleic acid showed poor stability during a frying cycle (Marangoni et al., 2020). Soybean oil with high linoleic acid content presented the higher oxidation rate. Consumption of oils high in monounsaturated fats is considered healthier (Thomsen et al., 1999). Decreasing the amount of polyunsaturated fatty acids, with low linoleic acid content, improves plant oil's oxidative stability and brings its acyl composition closer to that of olive oil. As a result, the cultivation of soybean varieties with low linoleic acid has become an important goal of high-quality soybean breeding (Do et al., 2019).

The linoleic acid biosynthesis is highly regulated, involving spatial separation of biosynthetic steps between different organelle compartments and the exquisite control of several biosynthetic steps by one or multiple biochemical mechanisms. Based on the next-generation sequencing technology and the genome wide association analysis (GWAS), the considerable research has gone into genetically reducing the level of linoleic acid, some key genes involved in the development of linoleic acid have identified (Li et al., 2019; Singh et al., 2001; Sivaraman et al., 2004). For instance, FAD2 is a key gene encoding an ER membrane-bound FA desaturase 2, which catalyzes the conversion of oleic acid to linoleic acid (Lakhssassi et al., 2017; Wen et al., 2018). RNA interference (RNAi) of D12 fatty acid desaturase (FAD2) and b-ketoacyl-acyl carrier protein synthase (KASII) decreased the accumulation of linoleic acids (J. Yang et al., 2018). Fatty Acid Desaturase-3 (FAD3) genes have been characterized which are responsible for the conversion of linoleic fatty acid precursors (18:2) to linolenic fatty acid precursors (18:3) (Povkhova et al., 2020). A research showed that the loss of function mutations in FAD3 resulted in accumulation to $67 \%$ linoleic acid in seed oil (Held et al., 2019; Thapa et al., 2018). 
WRINKLED1 (WRI1) transcription factor has been identified as a key regulator of the carbon partitioning into oils and proteins (Kong et al., 2019), WR/1 encodes for a APETALA2/ethylene responsive element binding protein, which is involved in global regulation of key enzymes of carbon metabolism and fatty acid biosynthesis (Kong et al., 2020). Further studies showed that WRI1 plays a role in processes such as lipid assembly, storage, seed development and photosynthesis (Guo et al., 2020; Kuczynski et al., 2020; Tang et al., 2019). For example, overexpression of the maize ZmWR/1 expressed under the embryo preferred OLE promoter increased fatty acid content in maize kernels (X. Zhang et al., 2019). In addition, Introduction of a seedspecific expression cassette carrying the Arabidopsis transcription factor WRINKLED1 (AtWR/1) into soybean, led to seed oil with levels of palmitic acid up to approximately $20 \%$ (Vogel et al., 2019). Clearly WR/1 acts as a key regulator of oil biosynthesis, which when expression is perturbed, translates to changes in different kinds of fatty acids accumulation (Chen et al., 2018; Kong and $\mathrm{Ma}, 2018)$.

The genome wide association analysis (GWAS) presents a powerful tool to discover SNPs associated with complex traits (Svishcheva et al., 2012). GWAS has become an affordable and powerful tool for dissecting complex traits in soybean (Wang et al., 2020; Yu et al., 2019; Zeng et al., 2017). To date, GWAS has been performed for the dissection of soybean fatty acids (T. Zhang et al., 2019). In our study, we discovered a candidate gene GmWR/14 that was correlated with linoleic acid content by GWAS analyses in three years. GmWR/14 belongs to the plant WRI1 protein family, and then the overexpression plasmid DNA was transferred into the soybean cultivar Jinong38 (JN38) by Agrobacterium-mediated transformation. As a result, we produce novel soybean lines containing only $11 \%$ linoleic acid in the seed oil. The present study can serve as a good reference for future studies on high-quality soybean breeding.

\section{Results}

\section{Phenotypic identification of linoleic acid content in soybean seeds}

From 2018 to 2020, the different fatty acids content of 510 soybean lines was collected from three different regions and determined by NIRSTM DS 2500 (FOSS, Hillerod, Denmark) after harvesting (Fig. 1A). The linoleic acid content of soybean lines was analyzed by SPSS 22.0 software. The variation range of linoleic acid was $35.12 \%-71.28 \%$, the Standard Deviation (SD) of linoleic acid content of soybean seeds was 8.2, the linoleic acid content of seeds approached the normal distribution (Fig. 1B). The results indicated that the linoleic acid content in different soybean lines is significantly different, which accorded with the genetic law of quantitative traits. We used the multiple linear regression model to analyze the relationship between linoleic acid and oleic acid. According to the regression coefficients of standardized multiple linear regression model, the content of linoleic acid is significantly negatively correlated with the content of oleic acid (Fig. 1C). The correlation coefficients between oleic acid and linoleic acid was -0.750 .

\section{A new candidate gene related to linoleic acid was discovered by GWAS}

In our experiments, SLAF-seq technology was used to sequence soybean genomic DNA. A total of 2,423,512 SNP markers were obtained, the results of SNP distribution on chromosomes are shown in Fig. 1D. Based on the linoleic acid content of 510 soybean lines, the TASSEL software were used for GWAS. SNP markers significantly correlated with the linoleic acid were detected. More than $50 \%$ of the SNPs were located in the intergenic regions (stretch of DNA sequences located between genes). $4.98 \%$ of the SNP loci were located in the protein coding regions. The $6.34 \%$ of SNP markers were located in introns (Fig. 1E). The LD distance was set to $8.9 \mathrm{~kb}$, the Manhattan for linoleic acid content in the different years (2018-2020) are shown in Fig. 1F. In 2018, 17 candidate genes related to soybean oleic acid content were screened using genome-wide association analysis (Table S1), in 2019, 10 candidate genes were screened by genome-wide association analysis (Table S1), in 2020, 10 candidate genes were screened by genome-wide association analysis (Table S1). The functional prediction of candidate genes is also shown in Table.S1. The first promising candidate gene GmWR/14 (Glyma.04G116500.1) was detected by GWAS in three years (Fig. 1F). There is no functional report about the candidate gene in soybean database, according to Swissport annotation. A similar gene in Arabidopsis belongs to the plant WRI1 protein family.

The expression of candidate gene GmWRI14 in different tissues and developmental stages of soybean 
To gain further insights into the organ specific expression profiles of GmWR/14, the distribution of the GmWR/14 was investigated by northern blot analysis. The result showed the highest transcript abundance of GmWR/14 was found in soybean seed. In order to validate their association to linoleic acids content, the GmWR/14 was measured in different tissues (root, stem, leaf and seed). The lectin gene (GenBank: A5547-127) was used as reference gene. The qRT-PCR results showed that the candidate gene GmWR/14 in soybean seedlings was expressed in different tissues, but the relative expression was significantly different, ranging from 26.12 to 52.32 in seeds, from 0.87 to 4.62 in soybean leaves, from 11.12 to 28.25 in stems, and from 16.60 to 31.12 in roots (Table.S2A). As general conclusion we can say that the correlation coefficient between GmWR/14 and linoleic acid content is $-0.901 \sim-0.912(P<0.01)$ (Table. S2B). This result strongly indicates that the candidate gene GmWR/14 is closely related to the linoleic acid content, specifically showing a negative effect on the linoleic acid content.

\section{Generation and molecular characterization of transgenic soybean plants over-expressing GmWRI14}

The recombinant plasmid designated pCAMBIA3300-GmWRI14 was introduced into Agrobacterium tumefaciens strain (Fig. S1). About 480 soybean cotyledon calluses were subjected to transformation. Since the transformants developed by the transformation strategy used could be chimeras in the $T_{0}$-generation, screening of $T_{1}$-generation plants for the identification of putative transformants was essential. Southern blot assay was used to detect the presence and determine the copy number of the GmWR/14 gene in the selected 6 putative transgenic lines, one copy of GmWR/14 was detected in $\mathrm{T}_{0} / \mathrm{T}_{1}$ generation of each line (Fig. 2A, B), the full length original blot have be included in the additional files Figure S2. The fatty acid content of the $T_{2}$ generation transgenic soybean seeds was determined by a near-infrared grain analyser. The data reveled the increasing trend in total oil in $T_{2}$ seeds from plants grown under greenhouse conditions (Fig. 3D), however, a significant decrease in linoleic acid was detected. The linoleic acid content in the positive strain decreased from $52.10-11.42 \%$, the oleic acid content increased from 23.21-41.02\% compared to the content in the JN 38 recipient (Table S3). Meanwhile the linoleic acid content of the GmWR/14 transgenic lines were reduced under field conditions during 2021, the result indicated that GmWR/14 decreases linoleic acid content in soybean seed. The transgenic lines and control varieties were investigated under field conditions. Both the JN38 control variety and transgenic lines had white flowers, round leaves and grey hairs. There was no significant difference between the transgenic lines and control varieties. The grain size was significant different (Fig. 3D). GmWR/14 gene was highly up-regulated in transgenic soybean compared to JiNong28, and its expression has been found to be closely connected to oil accumulation in various soybean plant, it has been proved that overexpression of GmWR/14 could lead to an significant increase in oil content (Fig. 3D). Meanwhile the average value of the results of three times of 100 grain weight measurement showed that there was obvious difference in 100 grain weight due to the different density owing to different contents of linoleic acid (Table.S3).

\section{Reduction of linoleic acid triggered by GmWRI14 expression is due to down-regulation of soybean FAD2}

To understand the genetic underpinnings of linoleic acid variation in soybean seed, we firstly analyzed the differentially expressed genes between GmWR/14 transgenic soybeans and no-transgenic soybean using the RNA-Seq data. RNA from three biological replicates of 6 transgenic lines and control JN38 was sequenced, as a result, more than $5.1 \times 107$ clean reads were obtained for different soybean lines after removing low quality reads, and the error rate of clean reads range from 0.02-0.03. A total of 1542 differentially expressed genes (DEGs) was screened by transcriptomics, after Kyoto Encyclopedia of Genes and Genomes (KEGG) analysis, the functional information of DEGs contains graphical representations of cellular processes, such as linoleic acid metabolism, alpha-linolenic acid metabolism. In the linoleic acid metabolism, the differentially expressed gene Volcano Plot (DEGVP) analysis revealed 8 differentially expressed genes are associated with linoleic acid metabolism (Fig. 3A, B, $C_{\text {,). }}$. To identify the FAD2 genes present in soybean genome are down-regulated by GmWR/14, we analyzed the expression of $F A D 2$ genes in transgenic soybean using qRT-PCR to determine whether the expression of this gene was altered. The lectin gene (GenBank: A5547-127) was used as reference gene. As a result, the expression of GmFAD2-1 and GmFAD2-2b genes in transgenic soybean lines were reduced, the GmFAD2-1 gene was expressed in different tissues, but the relative expression was significantly lower, ranging from 1.33 to 5.31 in the transgenic soybean leaves, from 10.21 to 19.23 in stems, and from 17.32 to 23.22 in seeds (Table.1). meanwhile the relative expression of GmFAD2-2b gene was significantly lower, ranging from 3.14 to 5.12 in soybean stems, from 5.46 to 9.11 in leaves, and from 21.21 to 27.35 in seeds (Table.1). As general conclusion we can say that the correlation coefficient between GmFAD2-1 and WRI1 is -0.970-0.982 $(P<0.01)$, and the correlation coefficient 
between GmFAD2b and WR/1 is -0.880-0.814 $(P<0.01)$ (Table.1). This result strongly indicates that the candidate gene WR/1 plays a negative role in regulating the linoleic acid content in the seeds. The linoleic acid content triggered by WR/1 expression is due to down-regulation of a soybean FAD2 (GmFAD2-1and GmFAD2-2b).

\section{Discussion}

Linoleic acid is the shortest chain n-6 fatty acid and the most common polyunsaturated fatty acids (PUFA) in plant oils and can be present in commercial oils at levels $>50 \%$ (Whelan and Fritsche, 2013). Overall, $60-70 \%$ of fatty acids of soybean oil are unsaturated, mainly characterized by high linoleic acid contents which are responsible for low oil oxidative stability, high temperature causes oxidative polymerization of linoleic acid. soybean oil has a less than desirable fatty acid composition (Hammond et al., 2005). The low-linoleic acid soybeans had competitive agronomic yield potential and stable linoleic acid levels from crop year to crop year, and oxidative stability of the oil was sufficient to successfully replace some previous usage of partially hydrogenated oils.

WRINKLED1 (WRI1), an APETALA2 (AP2)-type transcription factor, has been shown to be required for the regulation of carbon partitioning into fatty acid synthesis in plant seeds (Cernac and Benning, 2004). In 2018, the introduction of Arabidopsis transcription factor WRINKLED1 (AtWR/1) into soybean plant, led to seed oil with levels of palmitate up to approximately $20 \%$ (Vogel et al., 2019). In another study consider that the close correlation between WR/1 and Stearoy-Acyl-Carrier-Protein Desaturase (SAD) expression suggests the regulatory role of WR/1 in oleic acid accumulation (An and Suh, 2015). In our study, a new soybean WRINKLED 1 transcription factor GmWR/14 associated with linoleic acid in soybean seed were detected by SLAFseq combined with GWAS. The GmWR/14 is closely related to the linoleic acid content, specifically showing a negative effect on the linoleic acid content in soybean seeds. In one study, with a AtWR/1 expression, the levels of stearic acid and oleic acid up to $12.1 \%$ and $67.7 \%$, the increases in stearic and oleic acid came with the concomitant reduction in palmitic acid and linoleic acid, the linoleic acid content went from $42.1 \pm 0.2 \%$ in the wild-type down to $4.6 \pm 0.3 \%$ in the transgenic events (Baud et al., 2009). In our study, the transgenic soybean lines harboring GmWR/14 displayed a decrease in linoleic acid by 5-fold compared to the notransgenic soybean line JN38. Under control of the seed specific napin promoter, the soybean GmWRI1 increased oleic leading to increased oil content in soybean (Nakagawa et al., 2020). Similar results were reported from our research, overexpression of GmWR/14 in soybean increased oil content in soybean seed. Meanwhile the transgenic AtWR/1 transcript were monitored. Among the gene selected for transcript monitoring decreased expression of GmFAD2-1and GmFAD2-2b genes is observed. To identify GmFAD2-1 and GmFAD2-2b genes present in soybean genome are down-regulated by GmWR/14, the qRT-PCR analyses were conducted. These results indicate that GmWR/14 down-regulates specifically GmFAD2-1 and GmFAD2-2b in transgenic events, leading to decreases in linoleic acid in soybean seeds.

Oils are subject to several reactions that lead to physical-chemical, sensory and nutritional deterioration (Aladedunye and Przybylski, 2009), the widespread limitation of soybean oil production is due to rapid rancidity development during storage of oil leading to off flavor generation and become unacceptable by consumers (Esfarjani et al., 2019; Robards et al., 1988), therefore it is indispensable to adopt a stabilization step to decrease polyunsaturated fatty acids levels in soybean seed. Meanwhile the color of transgenic soybean oil showed that there was obvious difference in density due to the different fatty acid content (Fig. 3D). In our work, the GWAS was used to find SNP markers correlated with oleic acid content. The candidate gene GmWR/14 were detected in different years. We introducing the GmWR/14 into soybean, translated to an oil with low linoleic, with discernable changes in total oil content under greenhouse or field environments. The transgenic soybean lines showed the requirement for both seed specific homologues of FAD2 in soybean to be down-regulated to achieve lower linoleic acid content was also verified. It is the first time that the WR/1 had been reported to be associated with linoleic acid content in soybean. Our results provide a basis for deciphering the mechanism underlying the determination of fatty acid composition.

Since $W R / 1$ has been shown to be required for the regulation of carbon partitioning into fatty acid (FA) synthesis in plant seeds, many studies to date have focused on identifying genes upregulated by WRI1, several target genes, including Arabidopsis hemoglobin 1 (AtGLB1), Glycerol-3-Phosphate Acyltransferase (GPAT) have been found. In our study, many rate-limiting genes involved in glycolysis, PPP, and FA synthesis and desaturation, such as SAD, BS, MCMT, TAL and FAD2, showed a expression correlation with GmWRI14 (Fig. 4). In A. thaliana, the WRINKLED transcription factor is known to activate genes that regulate 
fatty acid biosynthesis in seeds via binding the AW-box (Fig. 4). While these genes explain some facets of the WR/1 overexpression phenotype, they do not fully account for WR/1 function, suggesting that additional target genes remain to be discovered. Meanwhile, repressor functions of WR/1 have been poorly investigated. In this study, we demonstrated a transcriptional repressor function of GmWR/14, showing that GmWR/14 repressed the FAD2, which has not been reported in previous studies. Our results significantly improve understanding of the transcriptional control exerted by GmWR/14 (Figure 4). The lack of an AW-box-related cis element in the promoter of FAD2 suggests that GmWR/14-mediated regulation of this gene might be indirect. Thus, it is reasonable to speculate that another trans-acting factor (depicted as " $X$ " in Figure 4), which is specifically associated with repressed target genes, converts GmWR/14 into a repressor. Although we mainly focused on the selected target gene in the current study, many potentially interesting target genes are repressed by GmWR/14. An interesting topic for future studies would be testing the relative contribution of co-repressor functions of GmWR/14 in Arabidopsis thaliana models.

\section{Methods}

\section{Plant materials}

The 510 soybean materials provided by the Biotechnology Center of Jilin Agricultural University and Guangzhou University of Chinese Medicine, the soybean materials were planted in 2017-2018 at Changchun, China ( $\left.123.53^{\circ} \mathrm{E}, 23.84^{\circ} \mathrm{N}\right)$, in $2018-2019$ at Qingdao, China $\left(120.41^{\circ} \mathrm{E}, 36.39^{\circ} \mathrm{N}\right)$, in $2019-2020$ at Guangdong, China $\left(113.41^{\circ} \mathrm{E}, 23.31^{\circ} \mathrm{N}\right)$. A randomized complete block design was used. The field was divided into three blocks and those were subdivided into eight sections. After that we did natural drying, then seeds were threshed for linoleic acid determination. Based on the collection, the soybean of two groups were identified with obvious differences in linoleic acid content, and were named Group 1 and Group 2. The names of the soybean lines and the fatty acid content are shown in Table. S2.

\section{Determination of fatty acids in soybean Seeds}

The content of linoleic acid and other four fatty acids (stearic acid, palmitic acid, oleic acid and linolenic acid) in soybean seeds were determined by NIRSTM DS 2500 (FOSS, Hillerod, Denmark). SPSS version 22.0 software (SPSS Inc, Chicago, IL, USA) was used to calculate the correlation coefficient of fatty acid in soybean seeds.

\section{Genotyping of soybean germplasms}

Total genomic DNA was extracted from leaves of each soybean line using a CTAB method according to Murray \& Thompson (Murray and Thompson, 1980). The 510 soybean materials were genotyped by Specific-Locus Amplified Fragment Sequencing (SLAF-seq) and SNP molecular markers were developed. The sequencing service is supported by Beijing Biomarker Biotechnology company. DNA extraction is the first step in sequencing. SNP molecular markers are used for GWAS analysis and genetic evolutionary correlation analysis. The restriction endonuclease combination was Rsal-Haelll.

\section{Genome-wide association analysis (GWAS)}

Based on the SNP markers obtained by SLAF-Seq technology, the correlation values between SNP markers and oleic acid content were obtained. TASSEL software can calculate the $\mathrm{Q}$ matrix of sample population structure according to the $K$ matrix, and finally get a correlation value of each SNP maker. The results of each model of each trait were annotated based on the $10^{-6}$ level of significance. In this experiment, Manhattan map were constructed by using Haploview software (BROAD Inc, Chicago, USA). The Manhattan map was used to represent the correlation between genotype data and phenotypic data. In this study, the candidate genes were predicted by using Swiss-Prot and NR databases.

\section{Quantitative reverse transcription-PCR}

The total RNA was extracted using Eastep ${ }^{\circledR}$ Super total RNA extraction Kit (TaKaRa, USA), then cDNA synthesis was performed using a reverse transcription kit (Omega. USA). The qRT-PCR analysis was performed using a Bio-Rad CFX system (Amersham Biosciences, Little Chalfont, Buckinghamshire,UK). Gene-specific primer pairs P3: (5'- 
TTGCCTGTCTAGATCCACAGCTGGTACCGAT-3') and P4(5'-TTGTGACCTCGACCTATTGGCGTTACCAATT-3') were used to amplify GmWR/14. The lectin gene (GenBank: A5547-127) was used as the reference gene. The reference gene was amplified with primer pairs P5: (5'-GCACTTAAGATACTCTAGGTAC-3') and P6: (5'-CCACCTCCCTACTATCCATT-3'). The amplification reaction conditions were pre-denaturation at $95^{\circ} \mathrm{C}$ for $10 \mathrm{~min}$, denaturation at $95{ }^{\circ} \mathrm{C}$ for $10 \mathrm{~s}$, annealing at $53^{\circ} \mathrm{C}$ for $20 \mathrm{~s}$, extension at $72{ }^{\circ} \mathrm{C}$ for $15 \mathrm{~s}$, and the amplification reaction conditions of the gene $\mathrm{GmWR} / 14$ are pre-denaturation at $95^{\circ} \mathrm{C}$ for 10 min, denaturation at $95^{\circ} \mathrm{C}$ for $30 \mathrm{~s}$, annealing at $59^{\circ} \mathrm{C}$ for $30 \mathrm{~s}$, extension at $72{ }^{\circ} \mathrm{C}$ for $35 \mathrm{~s}, 35$ cycles, and extension at $72{ }^{\circ} \mathrm{C}$ for 10 min. Three biological replicates were used for each gene.

\section{Vector construction and plant transformation}

The 1,815-bp cDNA of the GmWR/14 gene from the Bacillus thuringiensis strain HBF-18was ligated into the BamHI-Sacl site of pCAMBIA3300 to place the coding region under the regulatory control of the $35 \mathrm{~S}$ promoter and nos terminator (Fig. S1). The recombinant plasmid was named pCAMBIA3300- GmWR/14, and then introduced into Agrobacterium tumefaciens strain LBA4404 using the freezing-thaw method (Holsters et al., 1978). We introduced GmWR/14 into the soybean cultivar JN38 (Approval number 2012010), the seed of JN38 possesses good agronomic characteristics with high linoleic acid content. The transformation process was divided into five sequential steps: bacterial inoculation, cocultivation, resting, selection and plant regeneration.

\section{RNA-seq library preparation and sequencing}

Plants samples were processed for total RNA extraction using Eastep® Super total RNA extraction Kit (TaKaRa, USA), RNA quality was checked using a Nanodrop 2000c (Thermo Scientific, Hudson, NH, US). RNA-seq library preparation and sequencing were performed using the protocols described previously (Kumar et al., 2012; Sultan et al., 2012; Zhong et al., 2011). Fold change for gene expression was calculated by normalizing $C_{t}$ values at each developmental stage against endogenous control (Gm $\beta$ actin:Gm15g05570) using the $2^{-\Delta \Delta C t}$ method (Livak and Schmittgen, 2001).

\section{Data analysis}

The phenotypic data was measured and recorded using Microsoft Excel 2020 software. each data with three replicates. Differential saliency analysis, analysis of variance, correlation analysis and descriptiveness were performed by using SPSS 19.0 (IBM Corp, Armonk, NY, USA) software (Livak and Schmittgen, 2001). The statistical significance at $P \leq 0.01$ was calculated. The positive and negative maps and histograms were constructed by using Graphpad Prism software (Graphpad Company, San Diego, CA).

\section{Abbreviations}

SLAF-seq: Specific-Locus amplified fragment sequencing; SNP: Single nucleotide polymorphism; qRT-PCR: Real-time quantitative PCR: LD: Linkage disequilibrium; GWAS: Genome-wide association study; cM: Centi morgan; QTL: Quantitative trait loci

\section{Declarations}

\section{Acknowledgements}

We are grateful for the generous grant from the Biotechnology Center of Jilin Agricultural University and the Jilin Academy of Agricultural Sciences that made this work possible. The project was funded by National Genetically Modified New Varieties of Major Projects of China (2019X8002-002). The funding bodies had no role in the design of the study and collection, analysis, and interpretation of data and in writing the manuscript.

\section{Availability of data and material}

The data that support the findings of this study are available from [Beijing Biomarker Biotechnology Co., Itd, Beijing, China] but restrictions apply to the availability of these data, which were used under license for the current study, and so are not publicly 
available. Data are however available from the authors upon reasonable request and with permission of [Beijing Biomarker Biotechnology Co., Itd, Beijing, China]. Other datasets supporting the conclusions of this article are included within the article and its additional files.

\section{Conflict of interests}

The authors declare that they have no competing interests.

\section{Authors' contributions}

QD designed the experiments. QD, XL planned and performed the experiments. Cristina Miceli ,QD edited the manuscript. All authors discussed the results and commented on the manuscript. All authors have read and approved the manuscript.

\section{References}

1. Aladedunye, F. A. and Przybylski, R. (2009). Degradation and nutritional quality changes of oil during frying. Journal of the American Oil Chemists' Society, 86(2), 149-156.

2. An, D. and Suh, M. C. (2015). Overexpression of Arabidopsis WRI1 enhanced seed mass and storage oil content in Camelina sativa. Plant Biotechnology Reports, 9(3), 137-148.

3. Baud, S.,Wuillème, S.,To, A.,Rochat, C. and Lepiniec, L. (2009). Role of WRINKLED1 in the transcriptional regulation of glycolytic and fatty acid biosynthetic genes in Arabidopsis. The Plant Journal, 60(6), 933-947.

4. Cernac, A. and Benning, C. (2004). WRINKLED1 encodes an AP2/EREB domain protein involved in the control of storage compound biosynthesis in Arabidopsis. The Plant Journal, 40(4), 575-585.

5. Chan, J. K.,Bruce, V. M. and McDonald, B. E. (1991). Dietary a-linolenic acid is as effective as oleic acid and linoleic acid in lowering blood cholesterol in normolipidemic men. The American journal of clinical nutrition, 53(5), 1230-1234.

6. Chen, L.,Zheng, Y.,Dong, Z.,Meng, F.,Sun, X.,Fan, X.,Zhang, Y. et al. (2018). Soybean (Glycine max) WRINKLED1 transcription factor, GmWRI1a, positively regulates seed oil accumulation. Molecular genetics and genomics, 293(2), 401-415.

7. Clemente, T. E. and Cahoon, E. B. (2009). Soybean oil: genetic approaches for modification of functionality and total content. Plant physiology, 151(3), 1030-1040.

8. De Groot, R. H.,Hornstra, G.,van Houwelingen, A. C. and Roumen, F. (2004). Effect of a-linolenic acid supplementation during pregnancy on maternal and neonatal polyunsaturated fatty acid status and pregnancy outcome. The American journal of clinical nutrition, 79(2), 251-260.

9. Do, P. T.,Nguyen, C. X.,Bui, H. T.,Tran, L. T.,Stacey, G.,Gillman, J. D.,Zhang, Z. J. et al. (2019). Demonstration of highly efficient dual gRNA CRISPR/Cas9 editing of the homeologous GmFAD2-1A and GmFAD2-1B genes to yield a high oleic, low linoleic and a-linolenic acid phenotype in soybean. BMC plant biology, 19(1), 1-14.

10. Esfarjani, F.,Khoshtinat, K.,Zargaraan, A.,Mohammadi-Nasrabadi, F.,Salmani, Y.,Saghafi, Z.,Hosseini, H. et al. (2019). Evaluating the rancidity and quality of discarded oils in fast food restaurants. Food science \& nutrition, 7(7), $2302-2311$.

11. Guo, W.,Chen, L.,Chen, H.,Yang, H.,You, Q.,Bao, A.,Chen, S. et al. (2020). Overexpression of GmWRI1b in soybean stably improves plant architecture and associated yield parameters, and increases total seed oil production under field conditions. Plant biotechnology journal, 18(8), 1639-1641.

12. Hammond, E. G.,Johnson, L. A.,Su, C.,Wang, T. and White, P. J. (2005). Soybean oil. Bailey's industrial oil and fat products.

13. Held, J. P.,Carrero-Colón, M. and Hudson, K. A. (2019). Combination of novel mutation in FAD3C and FAD3A for low linolenic acid soybean. Agrosystems, Geosciences \& Environment, 2(1), 1-4.

14. Holsters, M.,De Waele, D.,Depicker, A.,Messens, E.,Van Montagu, M. and Schell, J. (1978). Transfection and transformation of Agrobacterium tumefaciens. Molecular and General Genetics MGG, 163(2), 181-187.

15. Kong, Q. and Ma, W. (2018). WRINKLED1 transcription factor: How much do we know about its regulatory mechanism? Plant Science, 272, 153-156. 
16. Kong, Q.,Yang, Y.,Guo, L.,Yuan, L. and Ma, W. (2020). Molecular basis of plant oil biosynthesis: Insights gained from studying the WRINKLED1 transcription factor. Frontiers in plant science, 11, 24.

17. Kong, Q.,Yuan, L. and Ma, W. (2019). WRINKLED1, a "Master Regulator" in transcriptional control of plant oil biosynthesis. Plants, 8(7), 238.

18. Kuczynski, C.,McCorkle, S.,Keereetaweep, J.,Shanklin, J. and Schwender, J. (2020). An expanded role for WRINKLED1 metabolic control based on combined phylogenetic and biochemical analyses. BioRxiv.

19. Kumar, R.,Ichihashi, Y.,Kimura, S.,Chitwood, D. H.,Headland, L. R.,Peng, J.,Maloof, J. N. et al. (2012). A high-throughput method for Illumina RNA-Seq library preparation. Frontiers in plant science, 3, 202.

20. Lakhssassi, N.,Zhou, Z.,Liu, S.,Colantonio, V.,AbuGhazaleh, A. and Meksem, K. (2017). Characterization of the FAD2 gene family in soybean reveals the limitations of gel-based TILLING in genes with high copy number. Frontiers in plant science, $\mathbf{8}$, 324.

21. Li, H.,Thrash, A.,Tang, J. D.,He, L.,Yan, J. and Warburton, M. L. (2019). Leveraging GWAS data to identify metabolic pathways and networks involved in maize lipid biosynthesis. The Plant Journal, 98(5), 853-863.

22. Liu, X.,Qin, D.,Piersanti, A.,Zhang, Q.,Miceli, C. and Wang, P. (2020). Genome-wide association study identifies candidate genes related to oleic acid content in soybean seeds. BMC plant biology, 20(1), 1-14.

23. Livak, K. J. and Schmittgen, T. D. (2001). Analysis of relative gene expression data using real-time quantitative PCR and the 2- $\triangle \Delta C T$ method. methods, 25(4), 402-408.

24. Marangoni, F.,Agostoni, C.,Borghi, C.,Catapano, A. L.,Cena, H.,Ghiselli, A.,La Vecchia, C. et al. (2020). Dietary linoleic acid and human health: Focus on cardiovascular and cardiometabolic effects. Atherosclerosis, 292, 90-98.

25. Maskan, M. and Karataş, Ş. (1998). Fatty acid oxidation of pistachio nuts stored under various atmospheric conditions and different temperatures. Journal of the Science of Food and Agriculture, 77(3), 334-340.

26. Murray, M. and Thompson, W. F. (1980). Rapid isolation of high molecular weight plant DNA. Nucleic acids research, 8(19), 4321-4326.

27. Nakagawa, A. C.,Ario, N.,Tomita, Y.,Tanaka, S.,Murayama, N.,Mizuta, C.,Iwaya-Inoue, M. et al. (2020). High temperature during soybean seed development differentially alters lipid and protein metabolism. Plant Production Science, 23(4), 504512.

28. Povkhova, L.,Pushkova, E.,Dmitriev, A.,Kezimana, P.,Novakovskiy, R.,Melnikova, N.,Rozhmina, T. et al. (2020). Molecular markers based on SNPS in FAD3 genes for determination of linolenic acid content in flax seed. Paper presented at the BIOINFORMATICS OF GENOME REGULATION AND STRUCTURE/SYSTEMS BIOLOGY (BGRS/SB-2020).

29. Robards, K.,Kerr, A. F. and Patsalides, E. (1988). Rancidity and its measurement in edible oils and snack foods. A review. Analyst, 113(2), 213-224.

30. Singh, S.,Thomaeus, S.,Lee, M.,Stymne, S. and Green, A. (2001). Transgenic expression of a $\Delta 12$-epoxygenase gene in Arabidopsis seeds inhibits accumulation of linoleic acid. Planta, 212(5), 872-879.

31. Sivaraman, I.,Arumugam, N.,Sodhi, Y. S.,Gupta, V.,Mukhopadhyay, A.,Pradhan, A. K.,Burma, P. K. et al. (2004). Development of high oleic and low linoleic acid transgenics in a zero erucic acid Brassica juncea L.(Indian mustard) line by antisense suppression of the fad2 gene. Molecular Breeding, 13(4), 365-375.

32. Sultan, M.,Dökel, S.,Amstislavskiy, V.,Wuttig, D.,Sültmann, H.,Lehrach, H. and Yaspo, M.-L. (2012). A simple strand-specific RNA-Seq library preparation protocol combining the Illumina TruSeq RNA and the dUTP methods. Biochemical and biophysical research communications, 422(4), 643-646.

33. Svishcheva, G. R.,Axenovich, T. I.,Belonogova, N. M.,Van Duijn, C. M. and Aulchenko, Y. S. (2012). Rapid variance components-based method for whole-genome association analysis. Nature genetics, 44(10), 1166-1170.

34. Tang, T.,Du, C.,Song, H.,Aziz, U.,Wang, L.,Zhao, C. and Zhang, M. (2019). Genome-wide analysis reveals the evolution and structural features of WRINKLED1 in plants. Molecular genetics and genomics, 294(2), 329-341.

35. Thapa, R.,Carrero-Colón, M.,Addo-Quaye, C.,Held, J.,Dilkes, B. and Hudson, K. A. (2018). New alleles of FAD3A lower the linolenic acid content of soybean seeds. Crop Science, 58(2), 713-718. 
36. Thomsen, C.,Rasmussen, O.,Lousen, T.,Holst, J. J.,Fenselau, S.,Schrezenmeir, J. and Hermansen, K. (1999). Differential effects of saturated and monounsaturated fatty acids on postprandial lipemia and incretin responses in healthy subjects. The American journal of clinical nutrition, 69(6), 1135-1143.

37. Tompkins, C. and Perkins, E. G. (2000). Frying performance of low-linolenic acid soybean oil. Journal of the American Oil Chemists' Society, 77(3), 223-229.

38. Vogel, P. A.,Bayon de Noyer, S.,Park, H.,Nguyen, H.,Hou, L.,Changa, T.,Khang, H. L. et al. (2019). Expression of the Arabidopsis WRINKLED 1 transcription factor leads to higher accumulation of palmitate in soybean seed. Plant biotechnology journal, 17(7), 1369-1379.

39. Wang, L.,Yang, Y.,Zhang, S.,Che, Z.,Yuan, W. and Yu, D. (2020). GWAS reveals two novel loci for photosynthesis-related traits in soybean. Molecular Genetics \& Genomics, 295(3).

40. Wen, S.,Liu, H.,Li, X.,Chen, X.,Hong, Y.,Li, H.,Lu, Q. et al. (2018). TALEN-mediated targeted mutagenesis of fatty acid desaturase 2 (FAD2) in peanut (Arachis hypogaea L.) promotes the accumulation of oleic acid. Plant molecular biology, 97(1), 177-185.

41. Whelan, J. and Fritsche, K. (2013). Linoleic acid. Advances in Nutrition, 4(3), 311-312.

42. Yang, J.,Xing, G.,Niu, L.,He, H.,Guo, D.,Du, Q.,Qian, X. et al. (2018). Improved oil quality in transgenic soybean seeds by RNAimediated knockdown of GmFAD2-1B. Transgenic research, 27(2), 155-166.

43. Yang, L.,Cao, Y.,Chen, J.-N. and Chen, Z.-Y. (2009). Oxidative stability of conjugated linolenic acids. Journal of agricultural and food chemistry, 57(10), 4212-4217.

44. Yu, Z.,Chang, F.,Lv, W.,Sharmin, R. A.,Wang, Z.,Kong, J.,Bhat, J. A. et al. (2019). Identification of QTN and candidate gene for seed-flooding tolerance in soybean [Glycine max (L.) Merr.] using genome-wide association study (GWAS). Genes, 10(12), 957.

45. Zeng, A.,Chen, P.,Korth, K.,Hancock, F.,Pereira, A.,Brye, K.,Wu, C. et al. (2017). Genome-wide association study (GWAS) of salt tolerance in worldwide soybean germplasm lines. Molecular Breeding, 37(3), 1-14.

46. Zhang, T.,Wu, T.,Wang, L.,Jiang, B.,Zhen, C.,Yuan, S.,Hou, W. et al. (2019). A combined linkage and GWAS analysis identifies QTLs linked to soybean seed protein and oil content. International journal of molecular sciences, 20(23), 5915.

47. Zhang, X.,Hong, M.,Wan, H.,Luo, L.,Yu, Z. and Guo, R. (2019). Identification of key genes involved in embryo development and differential oil accumulation in two contrasting maize genotypes. Genes, 10(12), 993.

48. Zhang, Y.,He, J.,Wang, H.,Meng, S.,Xing, G.,Li, Y.,Yang, S. et al. (2018). Detecting the QTL-allele system of seed oil traits using multi-locus genome-wide association analysis for population characterization and optimal cross prediction in soybean.

Frontiers in plant science, 9, 1793.

49. Zhao, X.,Jiang, H.,Feng, L.,Qu, Y.,Teng, W.,Qiu, L.,Zheng, H. et al. (2019). Genome-wide association and transcriptional studies reveal novel genes for unsaturated fatty acid synthesis in a panel of soybean accessions. BMC genomics, 20(1), 116.

50. Zhong, S.,Joung, J.-G.,Zheng, Y.,Chen, Y.-r.,Liu, B.,Shao, Y.,Xiang, J. Z. et al. (2011). High-throughput illumina strand-specific RNA sequencing library preparation. Cold spring harbor protocols, 2011(8), pdb. prot5652.

\section{Tables}

Table.1 A Variance analysis of GmFAD2-1 and GmFAD2-2b expression in different tissues of transgenic soybean 


\begin{tabular}{|c|c|c|c|c|c|c|c|c|c|c|}
\hline \multirow[t]{2}{*}{ Gene } & \multirow[b]{2}{*}{$\begin{array}{l}\text { correlation } \\
\text { coefficient } \\
\text { with WRI1 }\end{array}$} & \multirow[t]{2}{*}{$\begin{array}{l}\text { Soybean } \\
\text { Name }\end{array}$} & \multicolumn{2}{|c|}{$\begin{array}{l}\text { relative expression } \\
\text { in leaves }\end{array}$} & \multicolumn{2}{|l|}{$\begin{array}{l}\text { relative } \\
\text { expression in } \\
\text { stem }\end{array}$} & \multicolumn{2}{|c|}{$\begin{array}{l}\text { relative expression } \\
\text { in roots }\end{array}$} & \multicolumn{2}{|c|}{$\begin{array}{l}\text { relative expression } \\
\text { in seed }\end{array}$} \\
\hline & & & mean & Sig. & mean & Sig & mean & Sig. & mean & Sig. \\
\hline \multirow{7}{*}{$\begin{array}{l}\text { GmFAD2- } \\
1\end{array}$} & & JN38 & $10.15 \pm 0.34$ & c & $36.11 \pm 0.42$ & b & $32.21 \pm 0.12$ & a & $35.11 \pm 0.25$ & a \\
\hline & & $\begin{array}{l}\text { JN38- } \\
\text { GmWRI14- } \\
1\end{array}$ & $5.31 \pm 0.24$ & $d$ & $14.23 \pm 0.21$ & c & $13.23 \pm 1.2$ & c & $19.23 \pm 0.54$ & c \\
\hline & $\begin{array}{l}-0.970- \\
-0.982\end{array}$ & $\begin{array}{l}\text { JN38- } \\
\text { GmWRI14- } \\
2\end{array}$ & $2.52 \pm 0.32$ & $d$ & $10.21 \pm 0.51$ & c & $23.23 \pm 0.12$ & b & $17.32 \pm 0.42$ & c \\
\hline & & $\begin{array}{l}\text { JN38- } \\
\text { GmWRI14- } \\
3\end{array}$ & $3.12 \pm 0.01$ & $d$ & $19.23 \pm 0.15$ & c & $29.11 \pm 0.51$ & b & $22.23 \pm 0.56$ & b \\
\hline & & $\begin{array}{l}\text { JN38- } \\
\text { GmWRI14- } \\
4\end{array}$ & $4.25 \pm 0.12$ & $d$ & $15.22 \pm 0.16$ & c & $16.23 \pm 0.23$ & c & $26.11 \pm 0.16$ & $b$ \\
\hline & & $\begin{array}{l}\text { JN38- } \\
\text { GmWRI14- } \\
5\end{array}$ & $3.22 \pm 0.02$ & $d$ & $18.25 \pm 0.31$ & c & $20.54 \pm 0.26$ & $b$ & $23.22 \pm 0.61$ & $b$ \\
\hline & & $\begin{array}{l}\text { JN38- } \\
\text { GmWRI14- } \\
6\end{array}$ & $1.33 \pm 0.01$ & $d$ & $18.22 \pm 0.25$ & c & $21.51 \pm 0.16$ & $b$ & $19.23 \pm 0.12$ & c \\
\hline \multicolumn{11}{|l|}{$\begin{array}{l}\text { GmFAD2- } \\
2 b\end{array}$} \\
\hline & & $\begin{array}{l}\text { JN38- } \\
\text { GmWRI14- } \\
1\end{array}$ & $6.23 \pm 0.21$ & $d$ & $3.14 \pm 0.17$ & $d$ & $11.95 \pm 0.54$ & c & $22.75 \pm 0.26$ & $b$ \\
\hline & $\begin{array}{l}-0.880- \\
-0.814\end{array}$ & $\begin{array}{l}\text { JN38- } \\
\text { GmWRI14- } \\
2\end{array}$ & $5.46 \pm 0.11$ & $d$ & $4.22 \pm 0.22$ & $d$ & $12.51 \pm 0.34$ & c & $21.21 \pm 0.13$ & b \\
\hline & & $\begin{array}{l}\text { JN38- } \\
\text { GmWRI14- } \\
3\end{array}$ & $6.45 \pm 0.15$ & $d$ & $4.42 \pm 0.2$ & $d$ & $11.11 \pm 0.54$ & c & $26.12 \pm 0.26$ & b \\
\hline & & $\begin{array}{l}\text { JN38- } \\
\text { GmWRI14- } \\
4\end{array}$ & $7.23 \pm 0.17$ & $d$ & $5.12 \pm 0.15$ & $d$ & $13.21 \pm 0.32$ & c & $25.15 \pm 0.65$ & b \\
\hline & & $\begin{array}{l}\text { JN38- } \\
\text { GmWRI14- } \\
5\end{array}$ & $7.51 \pm 0.22$ & $d$ & $4.78 \pm 0.25$ & $d$ & $9.51 \pm 0.26$ & d & $27.35 \pm 0.43$ & b \\
\hline & & $\begin{array}{l}\text { JN38- } \\
\text { GmWRI14- } \\
6\end{array}$ & $9.11 \pm 0.12$ & $d$ & $3.22 \pm 0.19$ & $d$ & $12.43 \pm 0.62$ & c & $26.21 \pm 0.22$ & $b$ \\
\hline
\end{tabular}

Note: The different uppercase letters indicate significant differences at $P<0.01$, the different lower letters indicate significant differences at $P<0.05$, as determined by Duncan's multiple-range test 
(A)

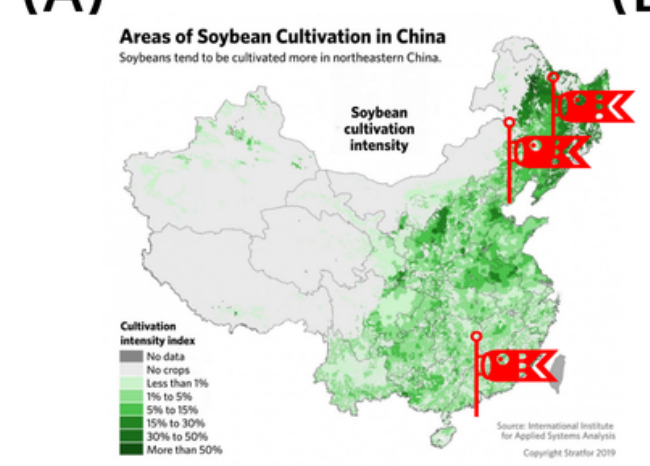

(c)

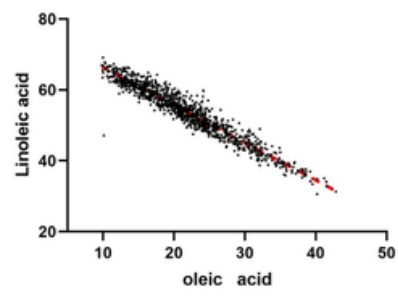

(E)

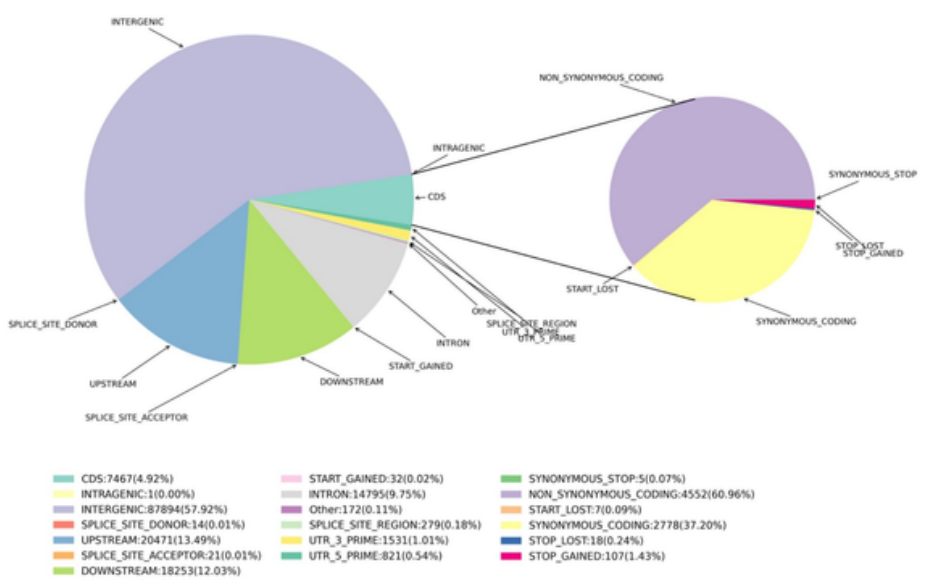

(D)
(F)
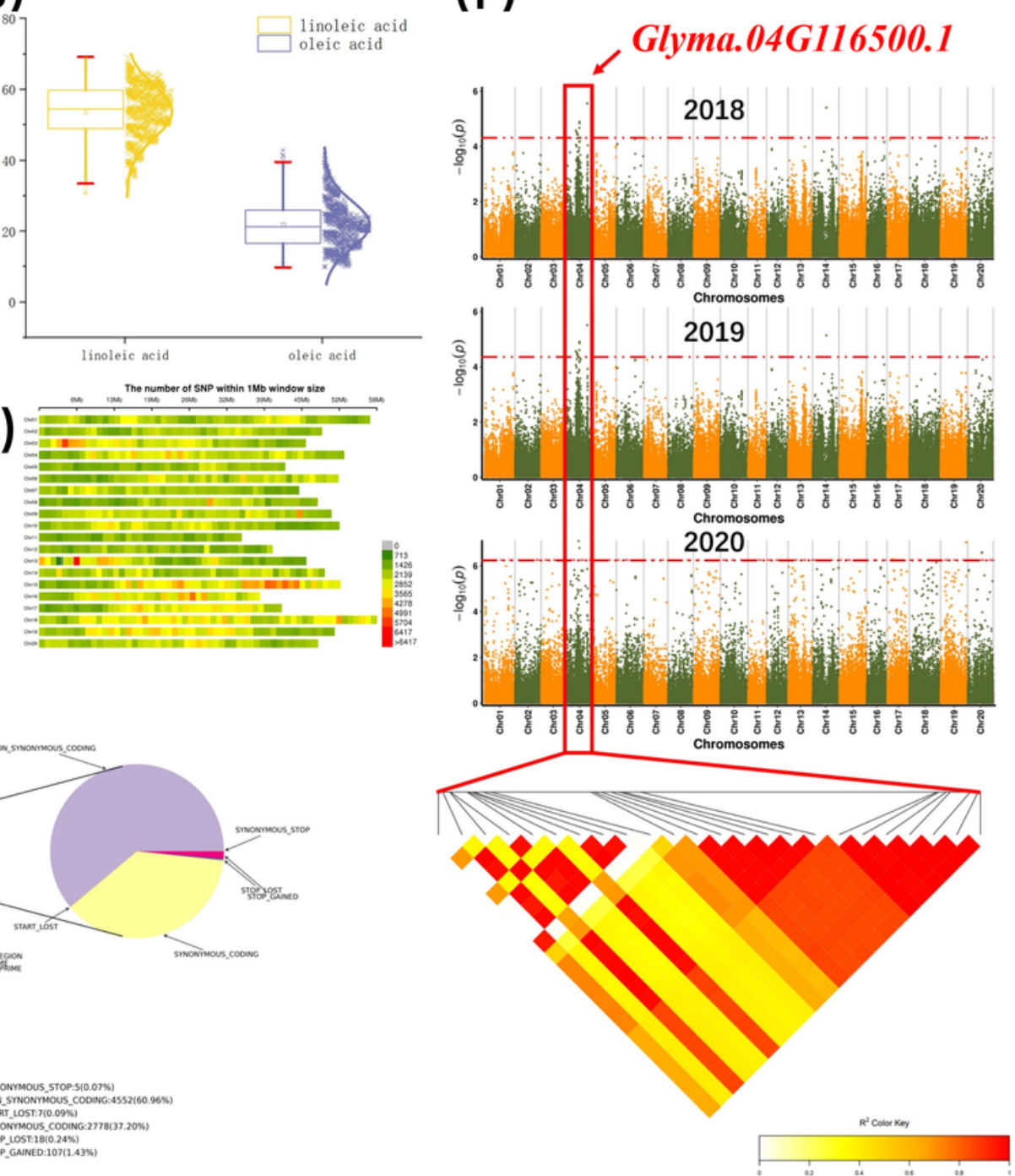

Figure 1

Phenotyping and genotyping of linoleic acid content in 510 Soybean germplasms. (A) 510 Soybean germplasms from China (Heilongjiang province, Shandong province and Guangdong province) were collected as the natural population. (B) The distribution of oleic acid content, the distribution resembles the bell-shaped curve for a normal distribution. (C) Association between percentages of oleic acid and linoleic acid, $y=-1.26 x+36.21$, is a linear equation, where y represents linoleic acid content and $x$ represent oleic acid content. (D) Distribution Map of SNPs on different chromosome. The abscissa is the length of the chromosome. Each band represents one chromosome. (E) Pie chart of SNPs Annotation. Left panel: SNPs percentages generally associated to genes. (F) Genome-wide Manhattan plots of associations for oleic acid content for 2018-2020 analysis. In the left panel, the X-axis indicates the SNPs along each chromosome; the Y-axis is the -log 10 (P-value) for the association. 


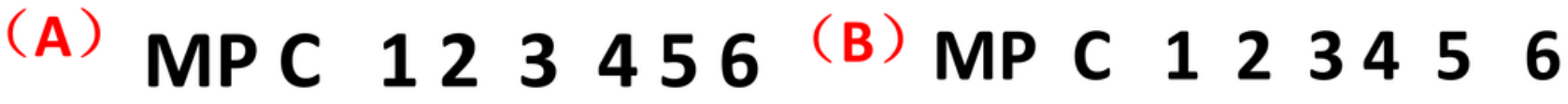

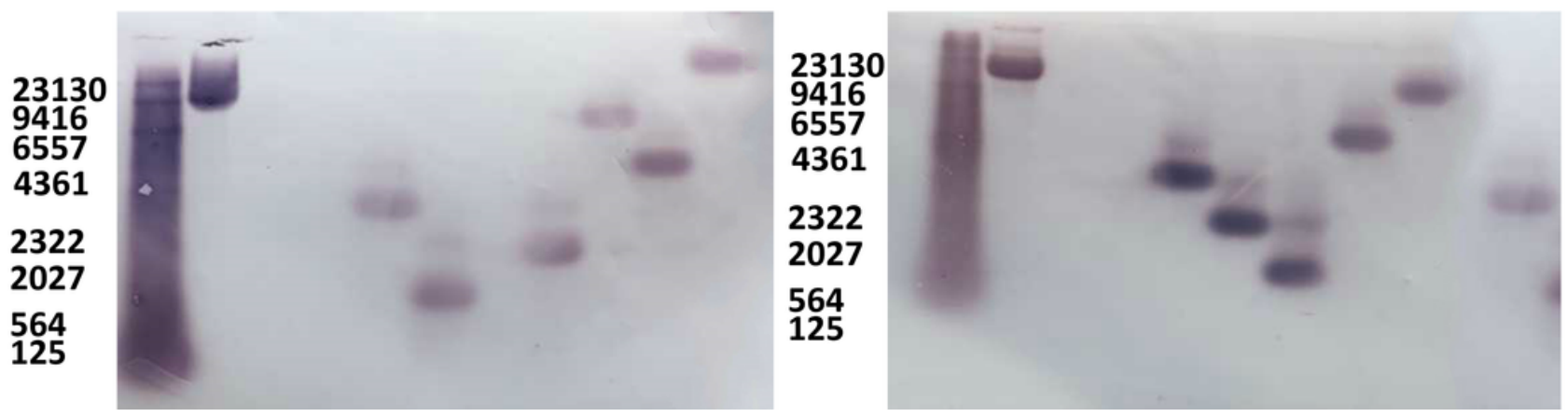

Figure 2

Verification of GmWRI14 expression in T0/T1 transgenic soybeans using Southern blot analysis. (A) Southern blot analysis of the copy number of the GmWRI14 expression cassette in T0 plants. (B) Copy number of the GmWRI14 expression cassette in T1 plants. C: control JN38. M: marker. The full length original blot have be included in the additional files Figure S2.

(A)

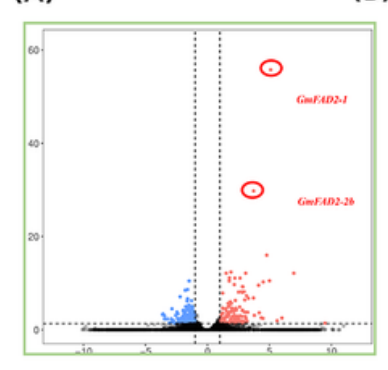

(c)

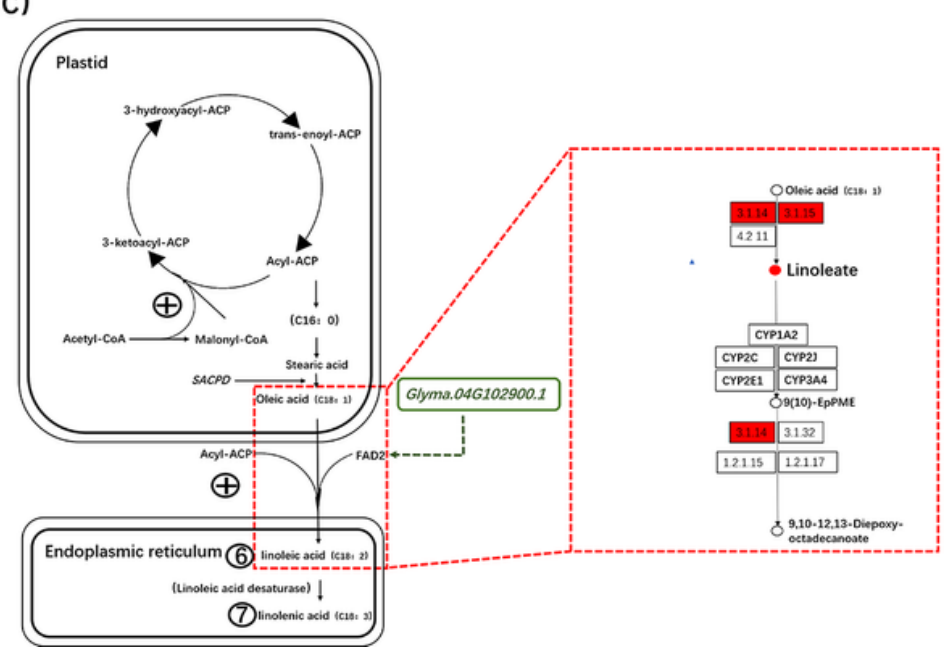

(B)

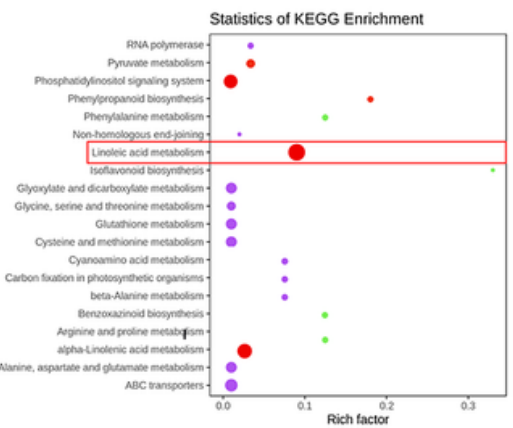

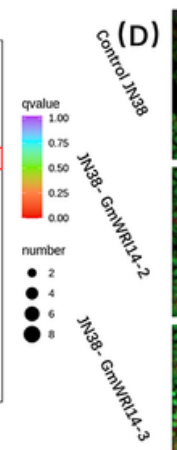

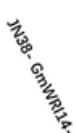

旁

意

藏

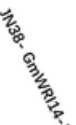

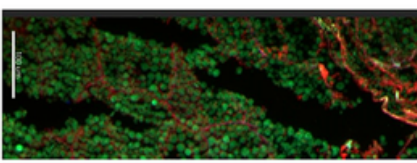
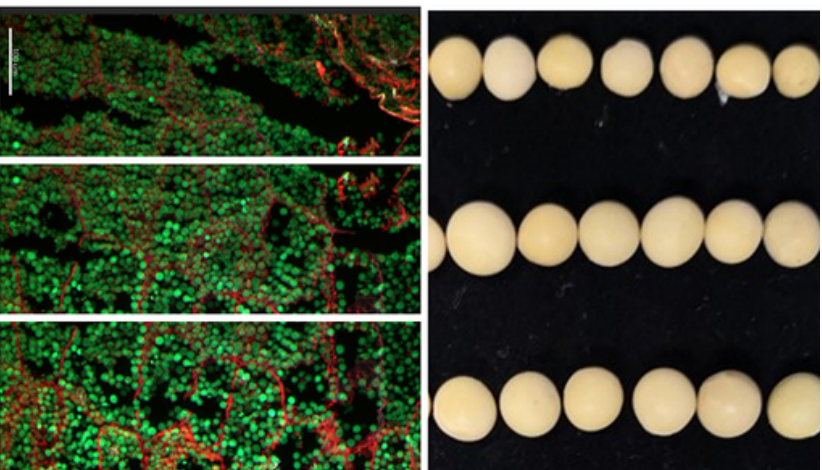

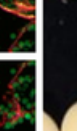
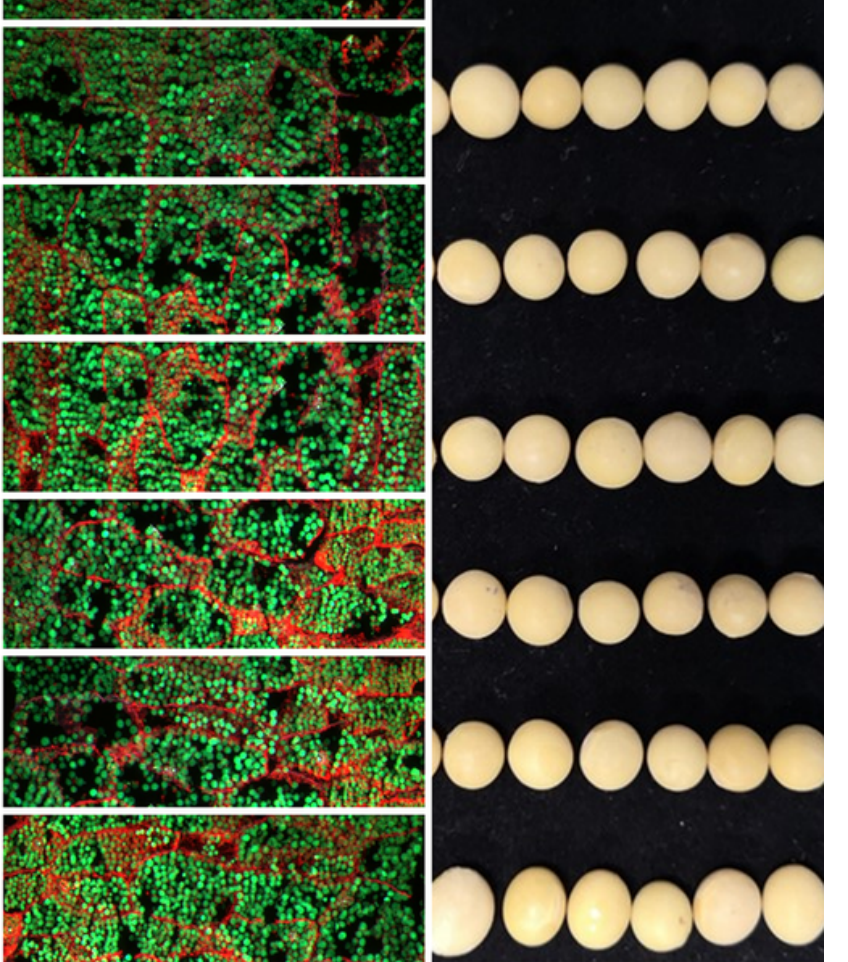

Figure 3

Analysis of different expression genes (DEGs) related to linoleic acid in GmWRI14 transgenic soybean plants. (A) KEGG analysis linoleic acid metabolism. Red indicated that the DEGs were down-regulation. (B) Functional characterization of GmWRI14 transgenic soybean for KEGG enrichment analysis. (C) DEGs of KEGG pathway map. For the GmWRI14 transgenic soybean, the enzyme labeled with red box was related to the down-regulated gene, The number in the box represents the fatty acid dehydrogenase (enzyme number), which explains the origin of phenotypic differences through the pathway. (D) Transmission electron microscopy pictures of seed cells from various soybean lines, The grain size of GmWRI14 transgenic soybean plants. The green region represents storage lipids. Scale bars,100um. 


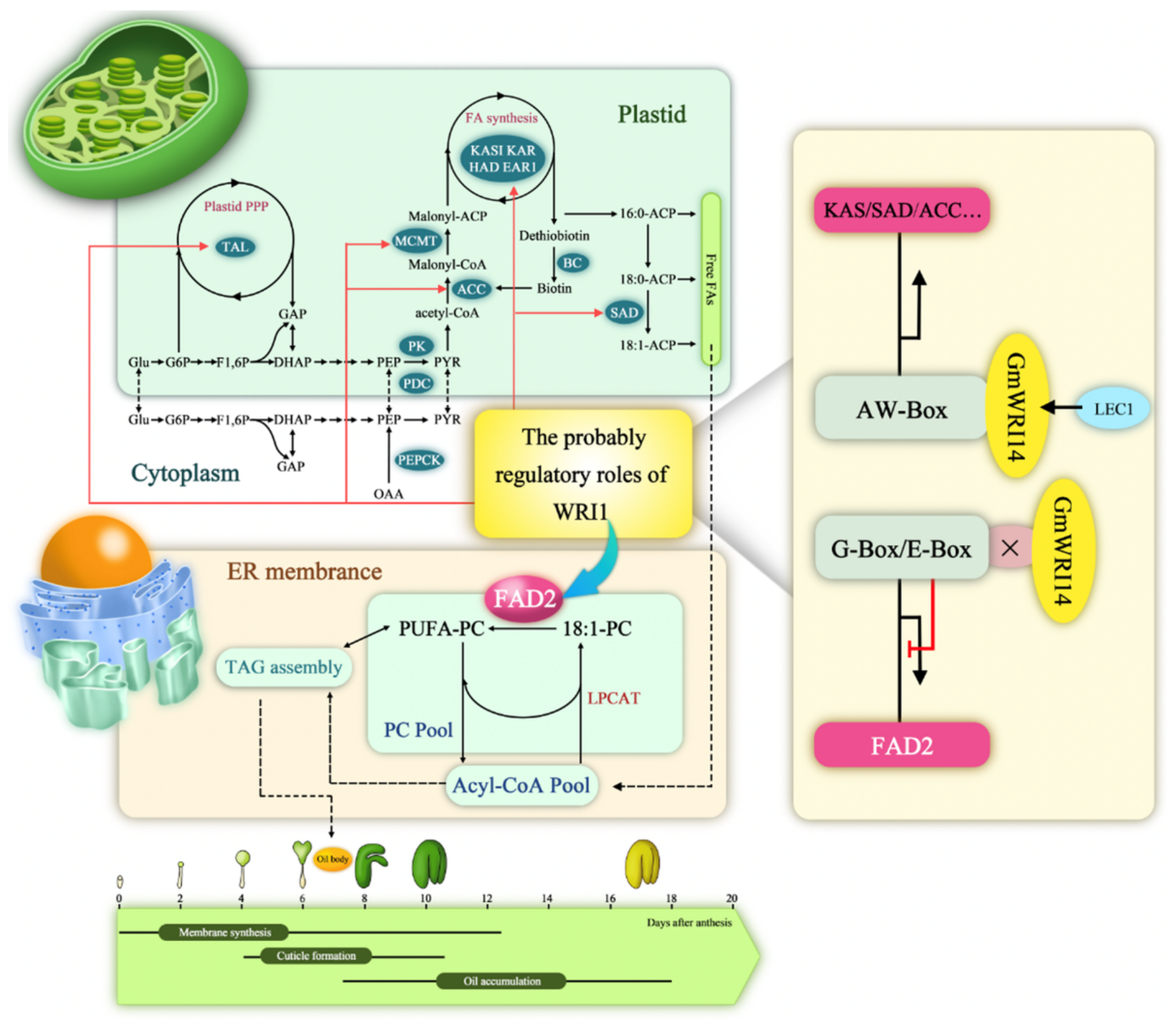

Figure 4

Model Depicting Transcriptional Control by GmWRI14. Red arrow lines represent the possible transcription regulation sites of GmWRI14. Abbreviations: PEPCK, phosphoenolpyruvate carboxykinase; SAD, stearoyl-ACP desaturase; BS, biotin synthase; KAS, ketoacyl-ACP synthase; EAR1, enoyl-ACP reductase 1; KAR, ketoacyl-ACP reductase; MCMT, malonyl-CoA; BC, biotin carboxylase; TAL, transaldolase; FAD2, fatty acid desaturase 2.

\section{Supplementary Files}

This is a list of supplementary files associated with this preprint. Click to download.

- FigureS1.png

- FigureS2.pptx

- Tables1.docx

- Tables2.docx 
- TableS3.docx

Page 15/15 